\title{
New Compact Antenna Based on Simplified CRLH-TL For UWB Wireless Communication Systems
}

\author{
Mohammad Alibakhshi-Kenari, ${ }^{1}$ Mohammad Naser-Moghadasi, ${ }^{1}$ \\ Ramazan Ali Sadeghzadeh, ${ }^{2}$ Bal Singh Virdee, ${ }^{3}$ Ernesto Limiti ${ }^{4}$ \\ ${ }^{1}$ Faculty of Eng, Science and Research Branch, Islamic Azad University, Tehran, Iran \\ ${ }^{2}$ Department of Elec. and Comp. Eng, K. N. Toosi University of Tech, Tehran, Iran \\ ${ }^{3}$ London Metropolitan University, Centre for Communications Technology, London N7 8DB, \\ United Kingdom \\ ${ }^{4}$ Dipartimento Di Ingegneria Elettronica, Università Degli Studi Di Roma Tor Vergata, Roma 00133, \\ Italy \\ Received 11 October 2015; accepted 30 November 2015
}

\begin{abstract}
This letter presents the experimental results of a novel planar antenna design which is synthesized using simplified composite left/right-handed transmissionline (SCRLH-TL), which is a version of a conventional composite left/right handedtransmission-lines (CRLH-TL), however, with the omission of shunt-inductance in the unit-cell. SCRLH-TL exhibits a right-handed response with nonlinear dispersion properties and a smooth Bloch-impedance distribution. Arranged within the inner slot of the antenna are three smaller rectangular patch radiators. Each patch radiator is embedded with an E-shaped notch, and located in the antenna conductor is a larger E-shaped notch next to the 50- $\Omega$ termination. The E-shaped notches constitute SCRLHTL property. The gap in the slot between the smaller patches and the conductor next to the larger E-shaped notch determines the impedance bandwidth of the antenna. The dimensions of the smaller patches determine the radiation characteristics of the antenna. The antenna is excited using a conductor-backed coplanar waveguide transmission-line. The antenna covers a bandwidth of $7.3 \mathrm{GHz}$ between $0.7 \mathrm{GHz}$ and $8 \mathrm{GHz}$, which corresponds to $167.81 \%$. In this band, the antenna resonates at $4.75 \mathrm{GHz}$ and $7 \mathrm{GHz}$; the gain and radiation efficiency at these frequencies are $4 \mathrm{dBi}-80 \%$ and $3.6 \mathrm{dBi}-73 \%$, respectively. The antenna's performance was validated through measurement. The antenna has dimensions of $0.0504 \lambda_{0} \times 0.0462 \lambda_{0} \times 0.0018 \lambda_{0}$, where $\lambda_{0}$ is freespace wavelength at $700 \mathrm{MHz}$. (c) 2016 Wiley Periodicals, Inc. Int J RF and Microwave CAE 00:000-000, 2016.
\end{abstract}

Keywords: patch antenna; ultra-wideband antenna; simplified composite right/left-handed transmission line (SCRLH); wireless communication systems; coplanar waveguide (CPW)

\section{INTRODUCTION}

Composite left/right handed (CRLH) transmission-lines (TLs) have been extensively investigated over the past several years [1]. Various microwave antennas have been

Correspondence to: M. Alibakhshi-Kenari; e-mail: makenari@ mtu.edu.

DOI: $10.1002 /$ mmce. 20956

Published online 00 Month 2016 in Wiley Online Library (wileyonlinelibrary.com) developed based on CRLH-TLs for wireless communications [2-5], which include techniques to enhance antenna bandwidth and radiation characteristics [6-8]. In addition, the emergence of the simplified composite left/righthanded transmission-line (SCRLH-TL) and its application in antenna design has resulted in antennas with wide bandwidth, good radiation patterns, and compact structures that can be easily fabricated using conventional techniques [9-11]. Unlike CRLH-TL, the SCRLH-TL resonates at zeroth and positive modes rather than

(C) 2016 Wiley Periodicals, Inc. 

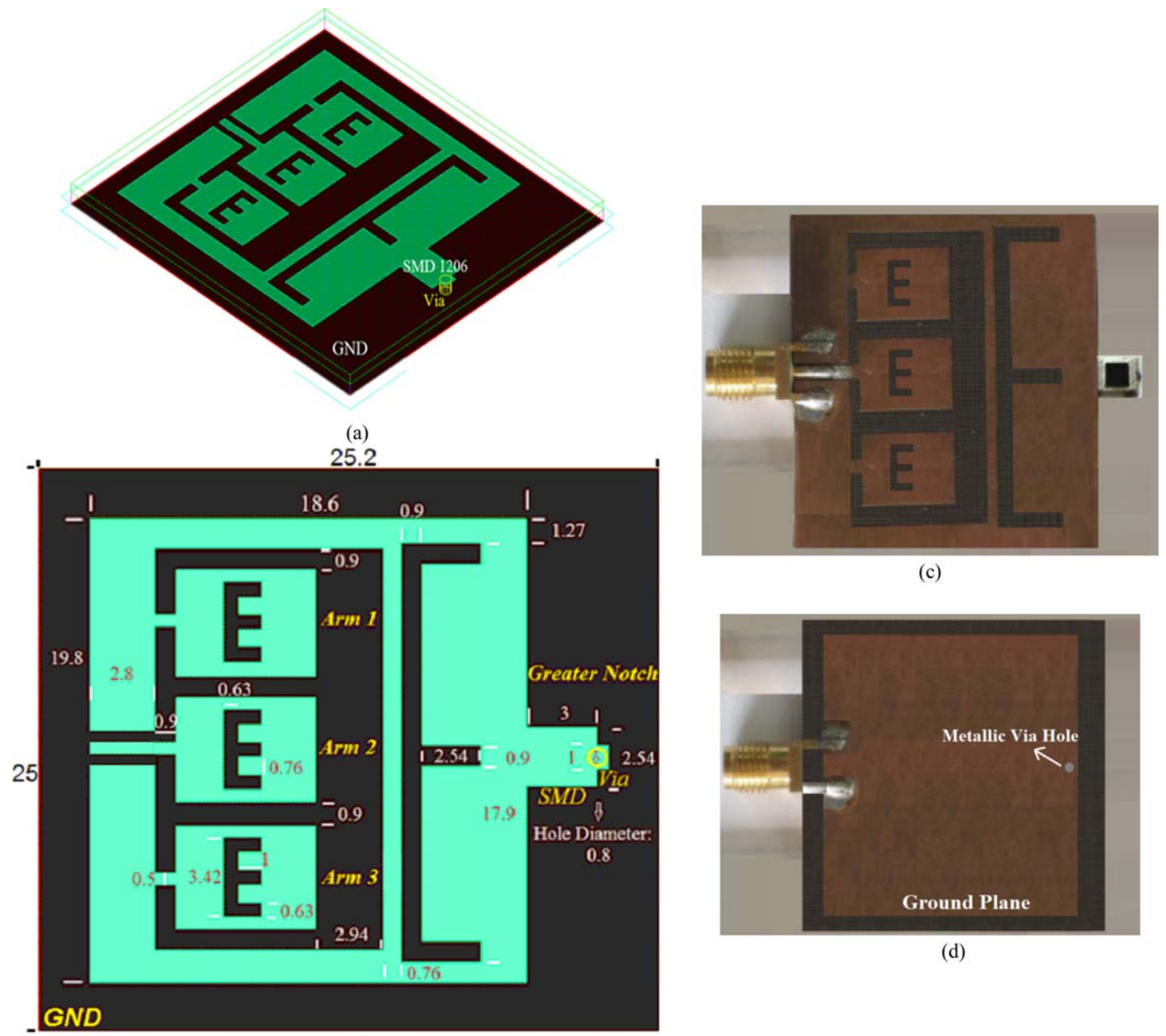

(c)

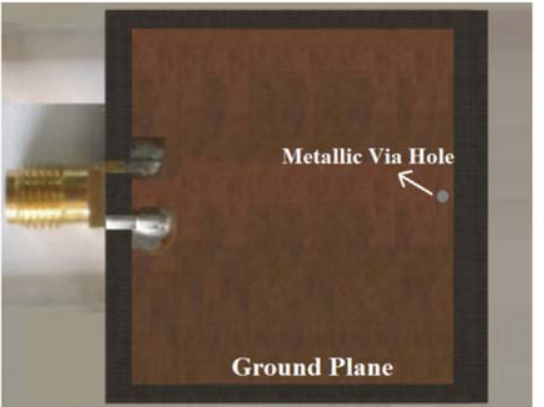

(d)

(b)

Figure 1 Test antenna prototype: (a) Isometric view, (b) Top view with dimensions (mm). Top view photograph, and (d) bottom view photograph.

negative order modes due to the absence of left-handed capacitance or inductance. When radiating at positive modes, the antenna achieves a higher efficiency than radiating at the zeroth and negative modes. This feature makes SCRLH-TL a good candidate for designing UWB antennas that have a small physical footprint.

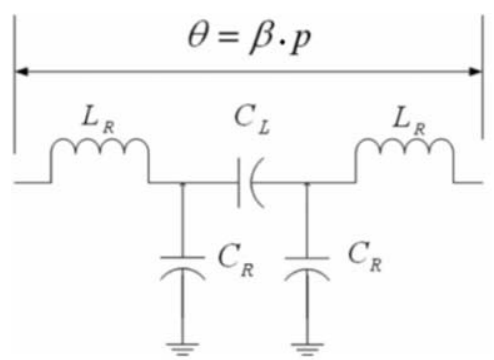

(a)

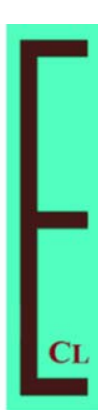

(b)
Figure 2 Simplified CRLH structure without $L_{\mathrm{L}}$ : (a) lumped structure, and (b) distributed equivalent one.
In this article, a new compact UWB antenna is proposed that is based on SCRLH-TL technology. The rectangular antenna comprises a slot within which are

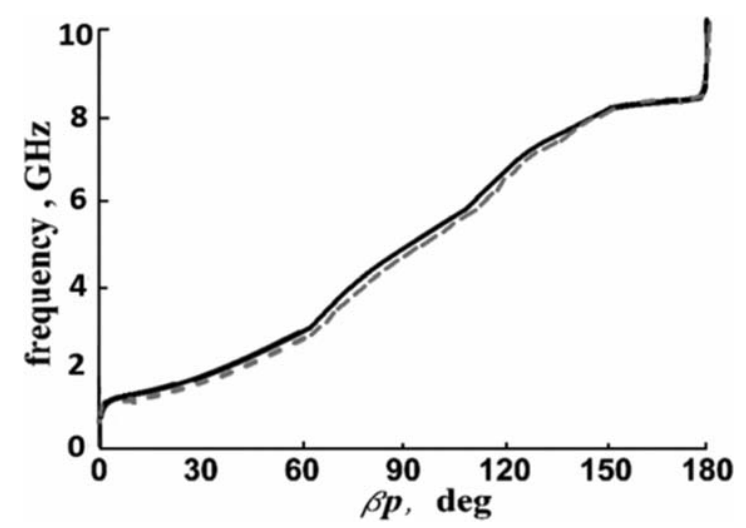

Figure 3 Dispersion diagram of the proposed antenna constructed using SCRLH-TL, computed by HFSS (solid-line) and Eq. (6) (dashed-line). 


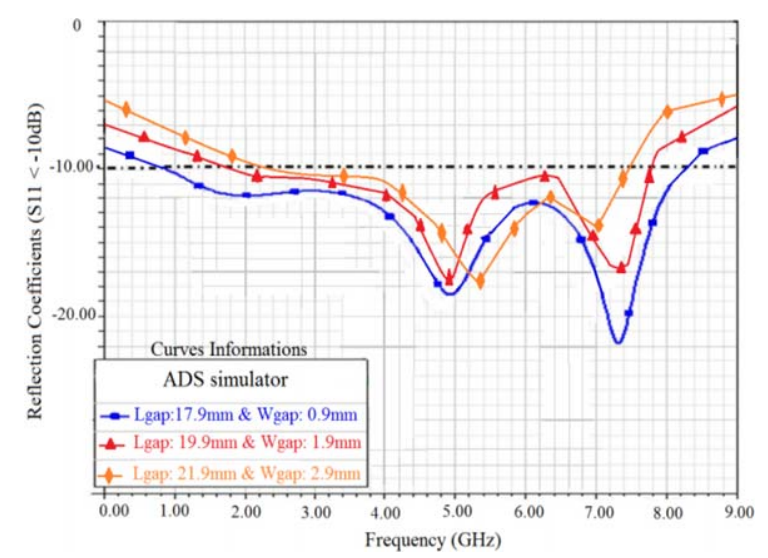

Figure 4 Parametric study of the length and width of big gap evidencing their impact on antenna impedance bandwidth.

included three rectangular radiating patches in a parallel arrangement. The patches include E-shaped notches. The antenna is fed through a conductor-backed coplanar feedline and is terminated in a $50-\Omega$ load that is shorted to the ground-plane using a via-hole. A larger E-shaped notch is embedded in the antenna in close proximity to the $50 \Omega$ termination. The dimensions of the three patches have a considerable influence on the radiation characteristics of the antenna. In addition, the slot gap between the inner patches and the outer conductor is analogous to a series left-handed capacitor $\left(C_{\mathrm{L}}\right)$, and it determines the impedance bandwidth of the antenna. The proposed antenna structure constitutes a simplified composite right/lefthanded transmission-line. Finally, the conductor-backed coplanar waveguide feed-line supports easy integration with wireless communication systems.

\section{ANTENNA STRUCTURE AND DESIGN APPROACH}

The configuration of the proposed rectangular antenna, shown in Figure 1, consists of an inner slot in which three smaller rectangular patches are included. Embedded on each patch is an E-shaped notch. The antenna is excited on the left-hand side through a conductor-backed coplanar waveguide (CPW) transmission-line, and the antenna is terminated on the right-hand side with a grounded $50-\Omega$ load. The resistive load minimizes unwanted reflections to improve the radiation characteristics of the antenna as shown in Section III. A larger E-shaped notch is included in the antenna next to the $50-\Omega$ load, which exhibits SCRLH-TL property as described in Ref. 9. The slot gap between the inner patches and the outer conductor, where the larger E-shaped notch is located, represents the lefthanded capacitance $\left(C_{\mathrm{L}}\right)$, so that, together with the smaller notches, it determines the impedance bandwidth of the antenna. Moreover, the number of smaller rectangular patches and their dimensions actually determine the antenna radiation characteristics. A sample antenna is fabricated on Rogers RO4003 substrate, with a thickness (h) of $0.8 \mathrm{~mm}$, dielectric constant $\left(\varepsilon_{\mathrm{r}}\right)$ of 3.38 and $\tan \delta$ of $22 \times 10^{-4}$.

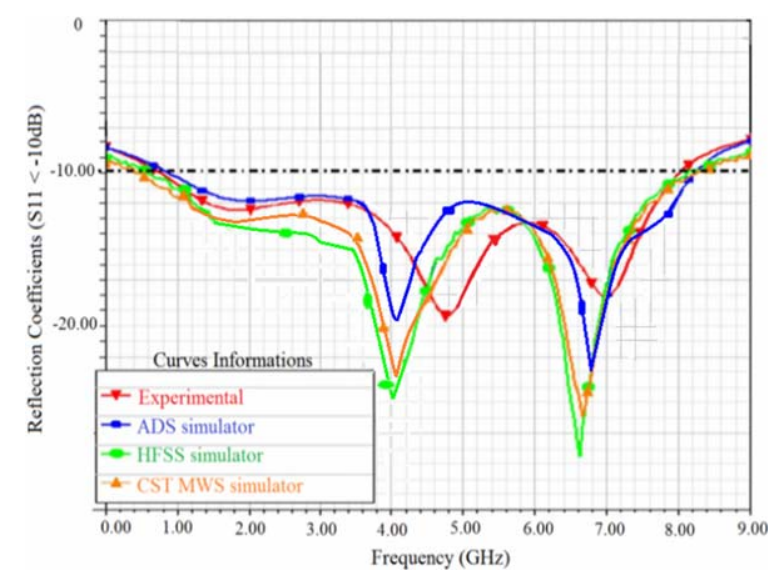

Figure 5 Antenna measured and simulated reflection coefficient response.

To verify the proposed antenna design and optimize the antenna structure three full wave simulators, i.e. of Agilent Advanced Design System (ADS), High Frequency Structure Simulator (HFSS), and CST Microwave Studio (CST-MWS) were employed. The simulation results were validated with the measured ones.

The configuration of the E-shaped notch was obtained via simulation analysis. As it will be shown later, the

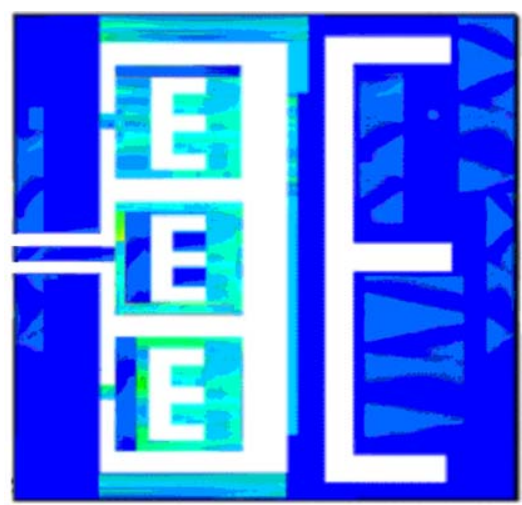

(a)

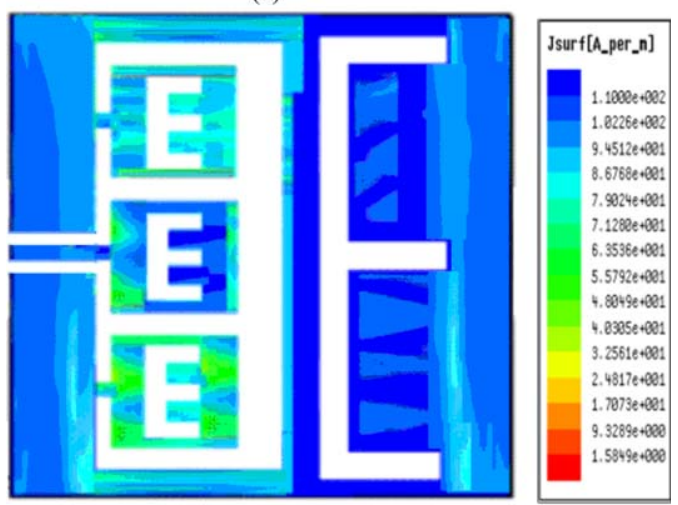

(b)

Figure 6 Current distribution over the antenna at (a) $4.75 \mathrm{GHz}$, and (b) $7 \mathrm{GHz}$. 


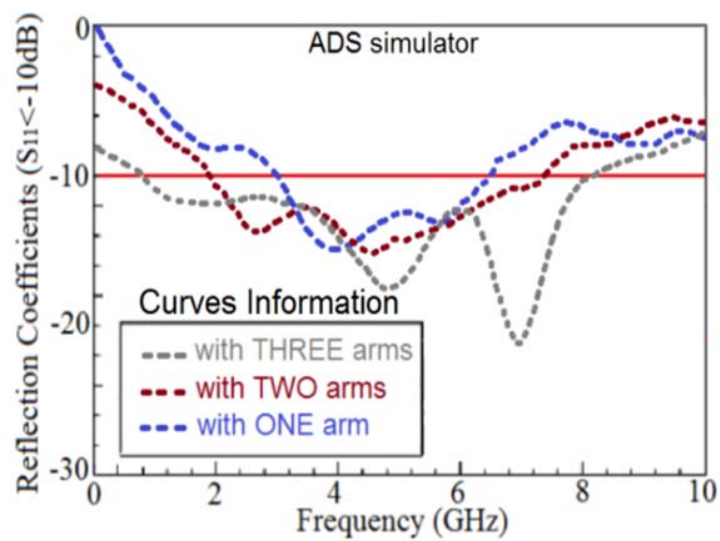

(a)

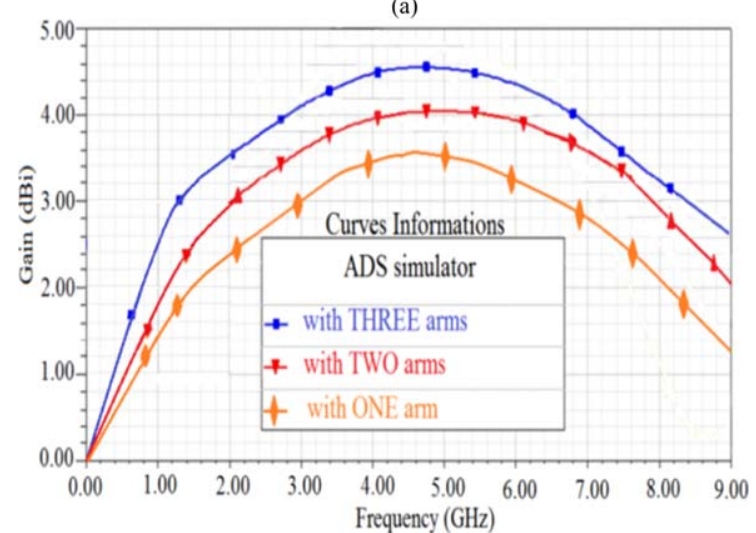

(b)

Figure 7 Simulated (ADS) antenna properties as a function of number of inner patch arms, (a) bandwidth, and (b) gain.

dimensions of the inner patch arms determine antenna gain and efficiency. The unit cell of proposed structure without left-handed inductance $\left(L_{\mathrm{L}}\right)$ and its corresponding distributed microstrip structure are shown in Figures $2 a$ and $2 b$, respectively. In Figure 2, the expressions relating lumped components and distributed microstrip parameters are given in Ref. 9, which are used to design the SCRLH structure. The expressions are here repeated for completeness:

$$
\begin{gathered}
L(\mathrm{nH})=0.2 l\left[\ln \left(\frac{1}{W+l}\right)+1.193+\frac{W+l}{3 l}\right] \\
\cdot\left(0.57-0.145 \ln \frac{W}{h}\right) \\
C(\mathrm{pF})=10 l\left[\frac{\sqrt{\varepsilon_{\mathrm{e}}}}{Z_{0}}-\frac{\varepsilon_{\mathrm{r}} W}{360 \pi h}\right] \\
\varepsilon_{\mathrm{e}}=\frac{\varepsilon_{\mathrm{r}}+1}{2}+\frac{\varepsilon_{\mathrm{r}}-1}{2} \sqrt{\frac{1}{\left(1+\frac{10 h}{W}\right)}}
\end{gathered}
$$

where $W$ and $l$ represent the width and length of the microstrip line, respectively; and $h$ and $\varepsilon_{\mathrm{r}}$ represent the thickness and relative permittivity of the substrate, respectively.

The E-shaped structure employed here is shown in Figure $2 \mathrm{~b}$. Based on the resonance frequencies of this structure the initial values of $C_{\mathrm{L}}, C_{\mathrm{R}}$, and $L_{\mathrm{R}}$ can be obtained from the dispersion relation of the SCRLH-TL. This is determined by applying the periodic boundary conditions related to the Bloch-Floquet theorem, given by:

$$
\begin{gathered}
\varnothing(\omega)=\beta(\omega) p=\cos ^{-1}\left(1+\frac{Z Y}{2}\right) \\
Z(\omega)=j\left(\omega L_{\mathrm{R}}-\frac{1}{\omega C_{\mathrm{L}}}\right) \\
Y(\omega)=j\left(\omega C_{\mathrm{R}}-\frac{1}{\omega L_{\mathrm{L}}}\right) \stackrel{\text { SCRLH-TL without } C_{\mathrm{L}} \text { or } L_{\mathrm{L}}, \text { Here, without } L_{\mathrm{L}}}{\longrightarrow} \\
Y(\omega)=j\left(\omega C_{\mathrm{R}}\right)
\end{gathered}
$$

where $Z$ and $Y$ represent the unit cell's series impedance and shunt admittance, respectively. $L_{R}$ is the series righthanded $(\mathrm{RH})$ inductance, and $\mathrm{C}_{\mathrm{R}}$ is the shunt $\mathrm{RH}$ capacitance. By substituting Eq. (4) and (5) into Eq. (3), the dispersion relation is given by:

$$
\varnothing(\omega)=\cos ^{-1}\left[1-\left(\frac{1}{2} \frac{C_{\mathrm{R}}}{C_{\mathrm{L}}}\left(\omega^{2} C_{\mathrm{L}} L_{\mathrm{R}}-1\right)\right)\right]
$$

Figure 3 shows the dispersion diagram of the antenna based on SCRLH-TL as determined by HFSS using Eq. (6).

In the SCRLH-TL structure, the negative order modes vanish with the absence of left-handed capacitance or inductance. Hence, the electrical size of the SCRLH-TL structure can be reduced by increasing $C_{\mathrm{L}}, L_{\mathrm{R}}$, and $C_{\mathrm{R}}$. This can be achieved by adjusting the structure dimensions. An optimization technique has been adopted within ADS, HFSS, and CST-MWS commercial tools, leading to the final size of the structure. The resulting antenna dimensions are annotated in Figure 1, and the corresponding values of the lumped elements are: $C_{\mathrm{L}}=8.2 \mathrm{pF}$, $L_{\mathrm{R}}=5.8 \mathrm{nH}$, and $C_{\mathrm{R}}=5.1 \mathrm{pF}$.

The antenna was then fabricated by using standard manufacturing techniques, and its performance measured. The physical dimensions of the antenna are: $21.6 \times 19.8 \times$ $0.8 \mathrm{~mm}^{3}$, and the corresponding electrical dimensions at $0.7 \mathrm{GHz}$ and $8 \mathrm{GHz}$, respectively, are: $504 \times 10^{-4} \lambda_{0} \times 462$ $\times 10^{-4} \lambda_{0} \times 18 \times 10^{-4} \lambda_{0}$ and $576 \times 10^{-3} \lambda_{0} \times 528 \times$ $10^{-3} \lambda_{0} \times 21 \times 10^{-3} \lambda_{0}$, where $\lambda_{0}$ is free-space wavelength. The ground-plane size of the antenna is $25.2 \times 25 \mathrm{~mm}^{2}$.

The efficiency of the antenna was measured in an anechoic chamber by feeding power to the proposed antenna and measuring the radiated electromagnetic field in the surrounding space. Efficiency was computed by taking the ratio of the radiated power to the input power

TABLE I Antenna Gain and Radiation Efficiency

\begin{tabular}{lccccc}
\hline & \multicolumn{2}{c}{ Simulation (ADS) } & & \multicolumn{2}{c}{ Measured } \\
\cline { 2 - 3 } $\begin{array}{l}\text { Freq. } \\
\text { (GHz) }\end{array}$ & $\begin{array}{c}\text { Gain } \\
(\mathrm{dBi})\end{array}$ & $\begin{array}{c}\text { Efficiency } \\
(\%)\end{array}$ & & $\begin{array}{c}\text { Gain } \\
(\mathrm{dBi})\end{array}$ & $\begin{array}{c}\text { Efficiency } \\
(\%)\end{array}$ \\
\hline 0.70 & 1.8 & 53 & & 1.2 & 50 \\
4.75 & 4.6 & 82 & & 4.0 & 80 \\
7.00 & 3.7 & 75 & & 3.6 & 73 \\
8.00 & 3.1 & 70 & & 3.1 & 68 \\
\hline
\end{tabular}




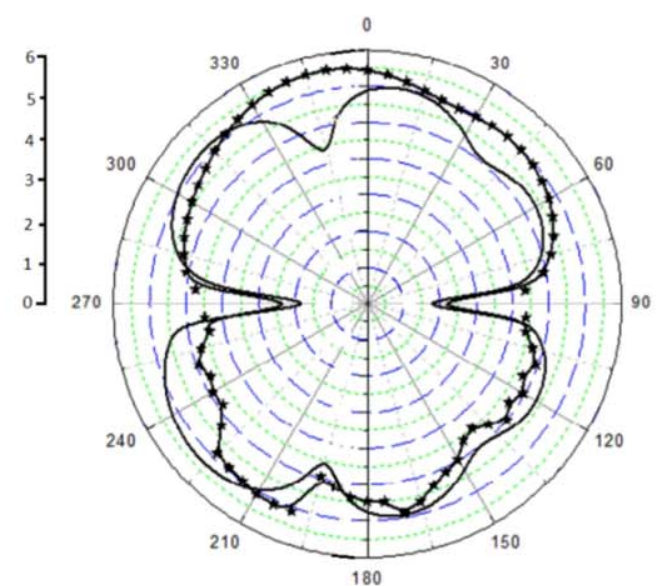

(a)

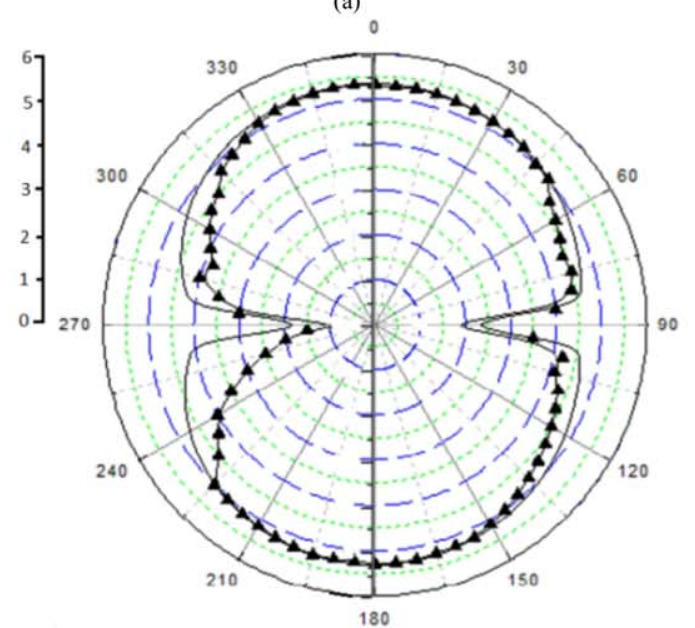

(b)
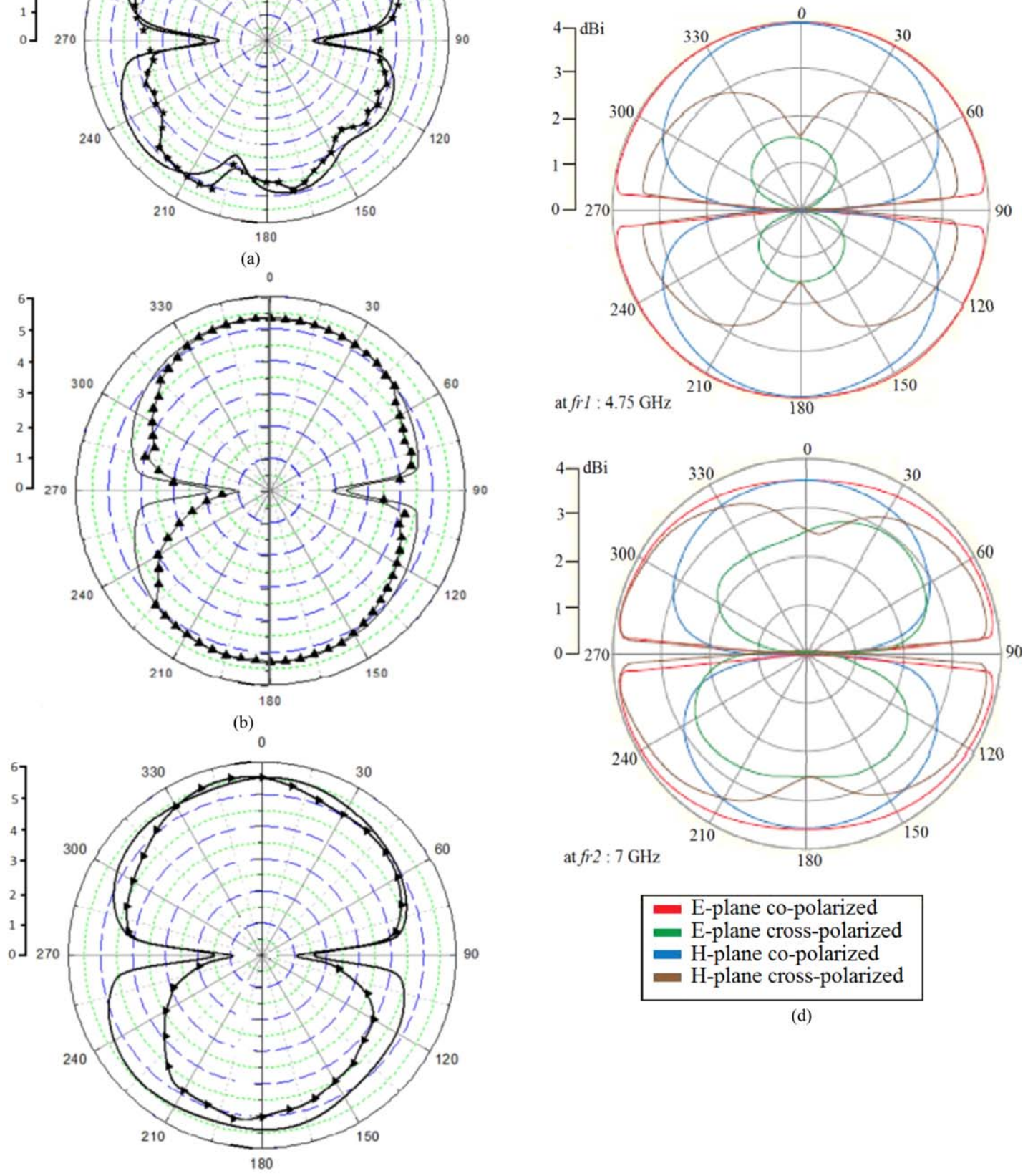

at $f r 2: 7 \mathrm{GHz}$

180

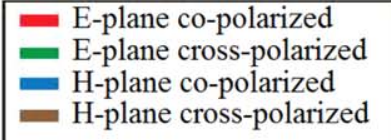

(d)

(c)

Figure 8 Simulated ( $E$-plane) and measured ( $E$-plane and $H$-plane) radiation patterns of the proposed antenna at the two resonance frequencies. (a) ADS at $f_{\mathrm{r} 1}=4.95 \mathrm{GHz}$ ( $*$ line) and $f_{\mathrm{r} 2}=7.33 \mathrm{GHz}$ (一 line), (b) CST-MWS at $f_{\mathrm{r} 1}=4.03 \mathrm{GHz}$ ( $\boldsymbol{\Lambda}$ line) and $f_{\mathrm{r} 2}=6.68 \mathrm{GHz}$ (一 line), (c) HFSS at $f_{\mathrm{r} 1}=4 \mathrm{GHz}\left(\boldsymbol{\Delta}\right.$ line) and $f_{\mathrm{r} 2}=6.6 \mathrm{GHz}$ ( - line), and (d) Measured at $f_{\mathrm{r} 1}=4.75 \mathrm{GHz}$ and $f_{\mathrm{r} 2}=7 \mathrm{GHz}$.

of the antenna. Antenna gain was measured by using the standard gain comparison technique, where pre-calibrated standard gain antenna was used to determine the absolute gain of the antenna under test.
The effect of the slot gap between the three patches and the outer conductor next to the larger E-shaped notch is shown in Figure 4. The simulation reveals that the slot's length and width have a major effect on the 


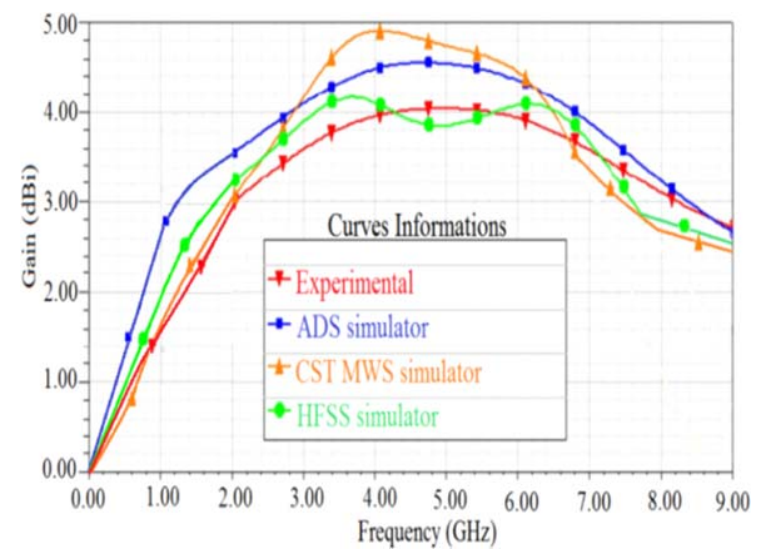

Figure 9 Antenna simulated and measured gain as a function of frequency.

antenna's impedance bandwidth. In fact, as it can be observed, the bandwidth improves significantly with a smaller length and width of the slot gap.

The measured and simulated reflection-coefficients of the proposed antenna are shown in Figure 5. The bandwidth criterion is based on the condition $S_{11}<-10 \mathrm{~dB}$. Three different simulation tools were used to determine the impedance bandwidth of the antenna. HFSS and CST are 3D EM simulators based upon different computational techniques. HFSS is based on Finite Element Method (FEM) while CST is based upon Finite Integration in Technique (FIT). However, ADS is a 2.5D EM simulator based upon Method of Moment (MoM). Simulation results from ADS predict the impedance bandwidth to be $7.44 \mathrm{GHz}(0.8 \mathrm{GHz}-8.24 \mathrm{GHz})$, while CSTMWS predicts a bandwidth of $7.88 \mathrm{GHz}(0.4 \mathrm{GHz}-$ $8.28 \mathrm{GHz}$ ), and finally HFSS estimates it to be $7.65 \mathrm{GHz}$ (0.55 GHz-8.2 GHz). As expected the correlation between HFSS and CST is excellent. The fractional bandwidth with ADS, CST-MWS, HFSS measurement are $164.6 \%, 181.6 \%, 174.9$, and $167.8 \%$, respectively. The measured impedance bandwidth is $7.3 \mathrm{GHz}(0.7$ $\mathrm{GHz}-8 \mathrm{GHz}$ ), which confirm the ultra-wideband operation of the antenna.

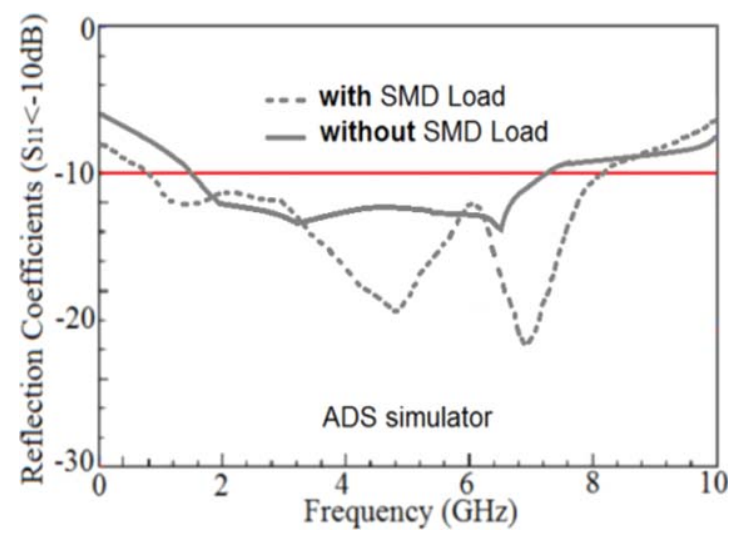

Figure 10 Antenna reflection-coefficient $\left(S_{11}\right)$ as a function of frequency with and without a $50-\Omega$ load.

Figure 5 clearly shows that the antenna exhibits two resonance frequencies at $4.15 \mathrm{GHz}$ and $6.8 \mathrm{GHz}$ (ADS), $4.03 \mathrm{GHz}$ and $6.68 \mathrm{GHz}$ (CST-MWS), and $4 \mathrm{GHz}$ and 6.6 GHz (HFSS), while the measured values are $4.75 \mathrm{GHz}$ and $7 \mathrm{GHz}$. Measured results in Figure 5 surprisingly show the antenna response to be closer to ADS simulation. This is attributed to errors in the manufacturing process employed to fabricate the antenna.

Current distributions of the antenna at its resonance frequencies of $4.75 \mathrm{GHz}$ and $7 \mathrm{GHz}$ are plotted in Figure 6. It is evident that current distributions are symmetric with respect to the symmetry line containing the feed.

The antenna is fed through a standard SMA connector that can cause an imbalance flow of current on the outer conductor of the antenna which can adversely affect the antenna's radiation properties. To circumvent such potential problems, three smaller patches have been introduced in the inner slot of the antenna. One of the three patches (middle) is excited, while the other two act as parasitic elements. These patches essentially concentrate the electromagnetic fields near antenna structure instead of spreading it over the antenna's ground-plane, which would otherwise contributes toward unwanted coupling thus degrading the overall performance of the antenna.

TABLE II Proposed Antenna Performance Compared to Reported Miniaturized UWB Antennas

\begin{tabular}{|c|c|c|c|c|}
\hline Papers & Dimensions@1 GHz & Bandwidth & Gain $(\max )$ & Efficiency (max) \\
\hline [12] 4UC antenna & $0.047 \lambda_{0} \times 0.021 \lambda_{0} \times 0.002 \lambda_{0} 14.2 \times 6.32 \times 0.8 \mathrm{~mm}^{3}$ & 1-3.2 GHz (104.8\%) & $2.3 \mathrm{dBi}$ & $62 \%$ \\
\hline [6] 5UC antenna & $0.056 \lambda_{0} \times 0.02 \lambda_{0} \times 0.005 \lambda_{0} 16.7 \times 7 \times 1.6 \mathrm{~mm}^{3}$ & 7.7-18.6 GHz $(82.9 \%)$ & $3.1 \mathrm{dBi}$ & $58 \%$ \\
\hline [12] 6UC antenna & $0.064 \lambda_{0} \times 0.021 \lambda_{0} \times 0.0027 \lambda_{0} 19.2 \times 6.32 \times 0.8 \mathrm{~mm}^{3}$ & $0.8-3.4 \mathrm{GHz}(123.8 \%)$ & $2.8 \mathrm{dBi}$ & $70 \%$ \\
\hline [6] 6UC antenna & $0.06 \lambda_{0} \times 0.02 \lambda_{0} \times 0.027 \lambda_{0} 18 \times 7 \times 0.8 \mathrm{~mm}^{3}$ & 7.5-16.8 GHz (74.4\%) & $2.1 \mathrm{dBi}$ & $44 \%$ \\
\hline [6] 7UC antenna & $0.072 \lambda_{0} \times 0.02 \lambda_{0} \times 0.005 \lambda_{0} 21.7 \times 7 \times 1.6 \mathrm{~mm}^{3}$ & $7.8-19.85 \mathrm{GHz}(87.2 \%)$ & $3.4 \mathrm{dBi}$ & $68 \%$ \\
\hline [6] 8UC antenna & $0.075 \lambda_{0} \times 0.02 \lambda_{0} \times 0.027 \lambda_{0} 22.6 \times 7 \times 0.8 \mathrm{~mm}^{3}$ & $7.25-17.8 \mathrm{GHz}(84.2 \%)$ & $2.3 \mathrm{dBi}$ & $48 \%$ \\
\hline [13] & $0.2 \lambda_{0} \times 0.05 \lambda_{0} \times 0.003 \lambda_{0} 60 \times 16 \times 1 \mathrm{~mm}^{3}$ & $0.67-2.55 \mathrm{GHz}(116.7 \%)$ & $4.74 \mathrm{dBi}$ & $62.9 \%$ \\
\hline [14] & $0.06 \lambda_{0} \times 0.06 \lambda_{0} \times 0.005 \lambda_{0} 18 \times 18 \times 1.6 \mathrm{~mm}^{3}$ & $1.8-2.35 \mathrm{GHz}(26.5 \%)$ & $3.69 \mathrm{dBi}$ & $20 \%$ \\
\hline [15] & $0.2 \lambda_{0} \times 0.017 \lambda_{0} \times 0.017 \lambda_{0} 60 \times 5 \times 5 \mathrm{~mm}^{3}$ & $0.8-2.5 \mathrm{GHz}(103 \%)$ & $0.45 \mathrm{dBi}$ & $53.6 \%$ \\
\hline [16] & $0.06 \lambda_{0} \times 0.06 \lambda_{0} \times 0.021 \lambda_{0} 18.2 \times 18.2 \times 6.5 \mathrm{~mm}^{3}$ & $1-2 \mathrm{GHz},(66.7 \%)$ & $0.6 \mathrm{dBi}$ & $26 \%$ \\
\hline [17] & $0.04 \lambda_{0} \times 0.04 \lambda_{0} \times 0.011 \lambda_{0} 12 \times 12 \times 3.33 \mathrm{~mm}^{3}$ & $2.34-2.54 \mathrm{GHz},(8.2 \%)$ & $1 \mathrm{dBi}$ & $22 \%$ \\
\hline [18] & $0.07 \lambda_{0} \times 0.08 \lambda_{0} \times 0.003 \lambda_{0} 20 \times 25 \times 0.8 \mathrm{~mm}^{3}$ & $3.45-3.75 \mathrm{GHz},(8.3 \%)$ & $2 \mathrm{dBi}$ & $27 \%$ \\
\hline Proposed antenna & $0.072 \lambda_{0} \times 0.066 \lambda_{0} \times 0.002 \lambda_{0} 21.6 \times 19.8 \times 0.8 \mathrm{~mm}^{3}$ & $0.7-8 \mathrm{GHz}(167.8 \%)$ & $4 \mathrm{dBi}$ & $80 \%$ \\
\hline
\end{tabular}

UC: unit cell. 
TABLE III Antenna Bandwidth With and Without $50-\Omega$ Load

\begin{tabular}{ll}
\hline Unloaded & $1.5-7.3 \mathrm{GHz}(\Delta f=5.8 \mathrm{GHz}) \approx 131.8 \%$ \\
Loaded & $0.8-8.24 \mathrm{GHz}(\Delta f=7.44 \mathrm{GHz}) \approx 164.6 \%$
\end{tabular}

Figure 7 shows that a larger number of patches can significantly improve the antenna's bandwidth and gain properties. In this plot, the antenna has been implemented on a Rogers RO4003 substrate with total dimensions of about $20 \times 20 \mathrm{~mm}^{2}$. Within this size, the maximum number of inner patch arms was restricted to three. The resulting antenna gain and radiation efficiency at its operating frequency range are given in Table I.

The radiation patterns of the antenna were simulated using ADS, CST-MWS, and HFSS. The simulated and measured radiation patterns are shown in Figure 8 at the two resonance frequencies.

At the two resonance frequencies, the antenna radiates in dipole-like pattern with a large coverage angle. The simulated and measured gains of the antenna are shown in Figure 9. The measured gain is higher than $1.2 \mathrm{dBi}$ between 0.7 and $9 \mathrm{GHz}$, with a maximum gain of $4.2 \mathrm{dBi}$ recorded at $4.8 \mathrm{GHz}$.

The proposed antenna, which is loaded with SCRLHTL unit cells, is compared with similar antennas reported to date in Table II. The proposed antenna exhibits the largest fractional bandwidth and highest efficiency. It has the second highest gain and its relatively small size is comparable to most previous works. These results clearly confirm the antenna is great candidate for ultra-wideband communication systems.

\section{PARAMETRIC STUDY ON $50 \Omega$ IMPEDANCE}

A. 50- $\Omega$ Load Effect on the Antenna Bandwidth

The antenna's reflection-coefficient with and without 50$\Omega$ load is shown in Figure 10. It can be observed from Figure 10 the $50-\Omega$ load improves the impedance matching and the impedance bandwidth from $131.8 \%$ (without $50 \Omega$ loading) up to $164.6 \%$ (with $50 \Omega$ loading). Excellent matching is observed close to the two resonance fre-

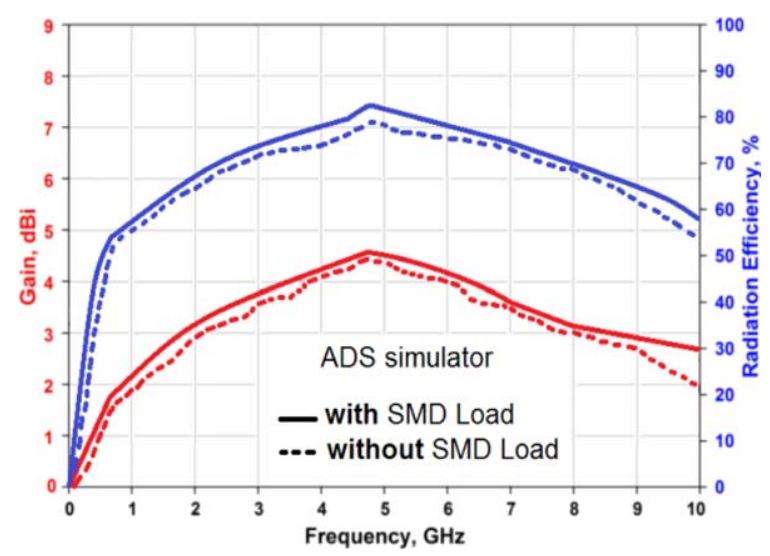

Figure 11 Antenna gain and efficiency with and without $50-\Omega$ $\mathrm{ohm}$ impedance loading simulated as a function of frequency.
TABLE IV Radiation Properties Unloaded/Loaded by $50 \Omega$

\begin{tabular}{lcccc}
\hline Unloaded & & & & \\
$\quad$ Frequency (GHz) & 0.7 & 4.75 & 7 & 8 \\
Gain (dBi) & 1.6 & 4.5 & 3.55 & 3 \\
$\quad$ Efficiency (\%) & 51 & 79 & 73 & 69 \\
Loaded & & & & \\
$\quad$ Frequency (GHz) & 0.7 & 4.75 & 7 & 8 \\
Gain (dBi) & 1.8 & 4.6 & 3.7 & 3.1 \\
Efficiency (\%) & 53 & 82 & 75 & 70 \\
\hline
\end{tabular}

quencies of the antenna, at 4.8 and $7 \mathrm{GHz}$, thus enabling effective radiation at the resonance frequencies. These results are presented in Table III.

\section{B. SMD Load Effect on the Radiation Performance}

Antenna gain and efficiency with and without $50 \Omega$ impedance loading are plotted in Figure 11 and tabulated in Table IV. These results show a small improvement is achieved with loading.

\section{CONCLUSIONS}

SCRLH-TL approach was used to develop a compact UWB antenna. SCRLH-TL structure was realized by including E-shaped notches in the radiating patches. Its radiative elements are contained within two regions: the first one is composed by three E-shaped notches organized as three cascaded arms that are carved on three radiation patches inside a gap area; the second region, containing the SCRLH- TL structure, is realized by a fourth E-notch with larger size, which is carved outside the gap area. A sample antenna with miniaturized dimension of $21.6 \times 19.8 \times$ $0.8 \mathrm{~mm}^{3}$ (with electrical dimensions $504 \times 10^{-4} \lambda_{0}$ by 462 $\times 10^{-4} \lambda_{0}$ by $18 \times 10^{-4} \lambda_{0}$ at $0.7 \mathrm{GHz}$ lower frequency and $576 \times 10^{-3} \lambda_{0}$ by $528 \times 10^{-3} \lambda_{0}$ by $21 \times 10^{-3} \lambda_{0}$ at $8 \mathrm{GHz}$ higher frequency) has been designed, manufactured, and tested. It operates over a wide bandwidth of $7.3 \mathrm{GHz}$ between $0.7 \mathrm{GHz}$ and $8 \mathrm{GHz}$, which corresponds to $167.81 \%$ fractional bandwidth. The antenna exhibits a minimum gain and radiation efficiency of $1.2 \mathrm{dBi}$ and $50 \%$, respectively, at $0.7 \mathrm{GHz}$ for integration in radiation systems for ultra-wideband applications. According to simulated and measured results, the proposed antenna with its small size demonstrates a very good potential for applications in ultra wideband wireless communication systems.

\section{REFERENCES}

1. C. Caloz, Metamaterial dispersion engineering concepts and applications, Proc IEEE 99 (2011), 1711-1719.

2. M. Alibakhshi-Kenari, M. Naser-Moghadasi, B.S. Virdee, A. Andújar, and J. Anguera, Compact antenna based on a composite right left handed transmission line, Microwave Opt Technol Lett 57 (2015), 1785-1788.

3. M. Alibakhshi-Kenari, M. Naser-Moghadasi, and R.A. Sadeghzadeh, Composite right-left-handed-based antenna with wide applications in very-high frequency-ultra-high frequency bands for radio transceivers, IET Microwaves Antennas Propag 9 (2015), 1713-1726. 
4. M. Alibakhshi-Kenari, M. Naser-Moghadasi, and R.A. Sadeghzadah, The resonating MTM based miniaturized antennas for wide-band rf-microwave systems, Microwave Opt Technol Lett 57 (2015), 1713-1726.

5. M. Alibakhshi-Kenari, M. Naser-Moghadasi, R.A. Sadeghzadeh, and B.S. Virdee, Metamaterial-based antennas for integration in UWB transceivers and portable microwave handsets, Int J. RF Microwave Comput Aided Eng 26 (2016), 88-96.

6. M. Alibakhshi-Kenari, Introducing the new wide band small plate antennas with engraved voids to form new geometries based on CRLH MTM-TLs for wireless applications, Int J Microwave Wireless Technol 6 (2014), 265-273.

7. M. Alibakhshi-Kenari and M. Naser-Moghadasi, Novel UWB miniaturized integrated antenna based on CRLH metamaterial transmission lines, AEUE Int J Electron Commun 69 (2015), 1143-1149.

8. M. Alibakhshi-Kenari, M. Naser-Moghadasi, and R.A. Sadeghzadah, Bandwidth and radiation specifications enhancement of monopole antennas loaded with split ring resonators, IET Microwaves Antennas Propag 9 (2015), 1487-1496.

9. X.Q. Lin, R.P. Liu, X.M. Yang, J.X. Chen, X.X. Ying, and Q. Cheng, Arbitrarily dual-band components using simplified structures of conventional CRLH TLs, IEEE Trans Microwave Theory Tech 54 (2006), 2902-2909.

10. J.Q. Gong and Q.X. Chu, Miniaturized microstrip bandpass filter using coupled SCRLH zeroth- order resonators, Microwave Opt Technol Lett 51 (2009), 2985-2989.
11. A.R. Raslan, A.A. Ibrahim, and A.M.E. Safwat, Resonanttype antennas loaded with CRLH unit cell, IEEE Antennas Wireless Propag Lett 12 (2013), 23-26.

12. M. Alibakhshi-Kenari, Printed planar patch antennas based on metamaterial, Int J Electron Lett 2 (2014), 37-42.

13. J. Luo, S. Gong, P. Duan, C. Mou, and M. Long, Small-size wideband monopole antenna with CRLH-TL for LTE mobile phone, Prog Electromagn Res C 50 (2014), 171-179.

14. M.A. Abdalla, A.A. Awad, and K.M. Hassan, Wide band high selective compact metamaterial antenna for $2 \mathrm{GHz}$ wireless applications, Antennas and Propagation Conf. 2014 Loughborough Antennas and Propagation Conference (LAPC 2014), 10-11 November 2014, Loughborough, Leicestershire, United Kingdom, pp. 350-354.

15. Y. Li, Z. Zhang, J. Zheng, and Z. Feng, Compact heptaband reconfigurable loop antenna for mobile handset, IEEE Antennas Wireless Propag Lett 10 (2011), 1162-1165.

16. C.J. Lee, K.M.K.H. Leong, and T. Itoh, Composite right/lefthanded transmission line based compact resonant antennas for RF module integration, IEEE Trans Antennas Propag 54 (2006), 2283-2291.

17. C.J. Lee, K.M.H. Leong, and T. Itoh, Broadband small antenna for portable wireless application, International Workshop on Antenna Technology: Small Antennas and Novel Metamaterials, iWAT 2008, 4-6 March 2008, pp. 10-13, Chiba-Japan.

18. C.-C. Yu, M.-H. Huang, L.-K. Lin, and Y.-T. Chang, A compact antenna based on MTM for WiMAX, Asia-Pacific Microwave Conference, (APMC), Macau-China, Dec. 2008.

\section{BIOGRAPHIES}

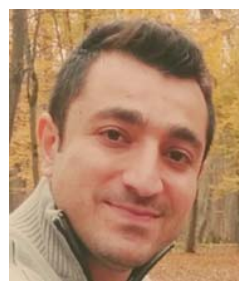

Mohammad Alibakhshi-Kenari was born in Freydonkenar, Iran on February 1988 He received the B.Sc. (Feb. 2010) and M.Sc. (Feb. 2013) degrees in Electrical Engineering and Telecommunication from the Islamic Azad University, Najafabad Branch of Esfahan, Iran and Shahid Bahonar University of Kerman, Iran, respectively. His research interests include microwave and millimeter wave circuits, transceivers, antennas and wave-propagation, CRLH-TLs, metamaterials, integrated RFtechnologies, embedded systems, electromagnetic-waves applications, and wireless telecommunication systems. He is now "Editor-inChief" in Journal Club for Electronic and Communication Engineering and member of the Applied Computational Electromagnetics Society (ACES). He also is a reviewer for several journals including IEEE TIE, IET MAP, OSA, Wiley, Elsevier, Taylor \& Francis, Springer and ACES. Mr. Alibakhshi has served as a Member of the Technical Program Committee (M-TPC) at IEEE international conferences. To date he has been published several journal papers.

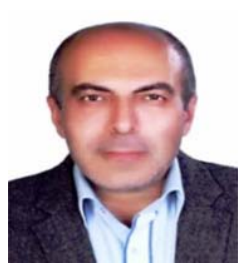

Mohammad Naser-Moghadasi was born in Iran, in 1959. He received the B.Sc. degree in Communication Engineering in 1985 from the Leeds Metropolitan University (formerly Leeds Polytechnic)-UK. Between 1985 and 1987 he worked as an RF-design-engineer for the Gigatech company in Newcastle Upon Tyne-UK. From 1987 to 1989 , he was awarded a full scholarship by the Leeds Educational Authority to pursue an M.Phil. on Studying CAD of microwave-circuits. He received his Ph.D. in
1993, from the University of Bradford-UK. He was offered then a two-years post-doc. at the University of Nottingham-UK, to pursue research on microwave cooking of materials. From 1995, Dr. Naser-Moghadasi joined Islamic Azad University, Science and Research Branch, Iran-Tehran, where he currently is head of postgraduate studies. His main areas of interest in research are microstrip antenna, microwave passive and active circuits, RF MEMS. Dr. Naser-Moghadasi is member of the IET, MIET, and IEICE. He has so far published over 150 papers.

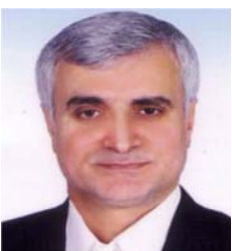

Ramazan Ali Sadeghzadeh received his B.Sc. in 1984 in Telecommunication Engineering from the Khajeh Nassir Toosi, University of Technology, Tehran-Iran, and M.Sc. in digital Communications Engineering from the University of Bradford and UMIST (University of Manchester, Institute of Science and Technology)-UK, as a joint program in 1987. He received his Ph.D. in electromagnetic and antenna from the University of Bradford-UK, in 1990. He worked as a Post-Doctoral Research assistant in the field of propagation, electromagnetic, antenna, Bio-Medical, and Wireless communications from 1990 till 1997. From 1984 to 1985 he was with Telecommunication Company of Iran (TCI) working on Networking. Since 1997 he is with K.N. Toosi University of Technology working with Telecommunications Department at faculty of Electrical and Computer Engineering. He has published more than 75 referable papers in international journals and conferences. Dr. Sadeghzadeh's current interests are numerical techniques in electromagnetic, antenna, propagation, radio networks, wireless communications, nanoantennas, and radar-systems. 


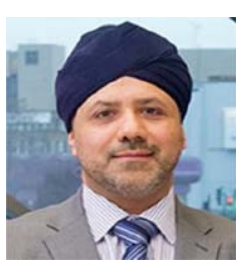

Bal Singh Virdee received the B.Sc. and MPhil degrees in CommunicationsEngineering from the University of Leeds-UK and his Ph.D. in ElectronicEngineering from the University of London-UK. He has worked in industry for various companies including Philips (UK) as an R\&D-engineer and FiltronicComponents Ltd. as a future products developer in the area of RF/microwave communications. He has taught at several academic institutions before joining London Metropolitan University where he is a Professor of MicrowaveCommunications in the Faculty of Life Sciences and Computing where he is the head of the Center for Communications Technology, and is the Director of London Metropolitan Microwaves. His research, in collaboration with industry and academia, is in the area of microwave wireless communications encompassing mobilephones to satellite-technology. Prof. Virdee has chaired technical sessions at IEEE international conferences and published numerous research-papers. He is Executive-Member of IET's Technical and Professional Network Committee on RF/Microwave-Technology. $\mathrm{He}$ is a FIET and a Senior-MIEEE.

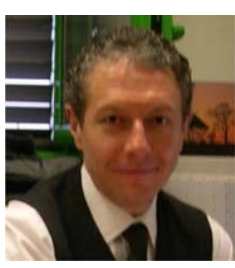

Ernesto Limiti is a full professor of Electronics in the Engineering Faculty of the University of Roma Tor Vergata since 2002, after being research and teaching assistant (since 1991) and associate professor (since 1998) in the same University. His research-activity is focused on three main-lines, all of them belonging to the microwave/millimetre-wave electronics area. The first one is related to characterization and modeling for active and passive microwave and millimetre-wave devices. Regarding active-devices, the research-line is oriented to the smallsignal, noise and large signal modelling as well as devices on diamond substrates. Novel methodologies have been developed and equivalent-circuit modeling strategies have been implemented both for small/large-signal operating regimes for different-device technologies. Design methodologies and characterization methods for low-noise devices and circuits are also in focus, as well as the analysis and design methodologies for linear/nonlinear microwavecircuits. The above research lines have produced more than 300 publications on refereed-international journals and presentations within international-conferences. 